\title{
Article
}

\section{Investigating optimum sample preparation for infrared spectroscopic serum diagnostics}

Lovergne, Lila, Clemens, Graeme, Untereiner, Valérie, Lukaszweski, Roman A., Sockalingum, Ganesh D. and Baker, Matthew James

Available at http://clok.uclan.ac.uk/12355/

Lovergne, Lila, Clemens, Graeme, Untereiner, Valérie, Lukaszweski, Roman A., Sockalingum, Ganesh D. and Baker, Matthew James ORCID: 0000-0003-23628581 (2015) Investigating optimum sample preparation for infrared spectroscopic serum diagnostics. Analytical Methods. ISSN 1759-9660 (Unpublished)

It is advisable to refer to the publisher's version if you intend to cite from the work. http://dx.doi.org/10.1039/C5AY00502G

For more information about UCLan's research in this area go to

http://www.uclan.ac.uk/researchgroups/ and search for <name of research Group>.

For information about Research generally at UCLan please go to http://www.uclan.ac.uk/research/

All outputs in CLoK are protected by Intellectual Property Rights law, including Copyright law. Copyright, IPR and Moral Rights for the works on this site are retained by the individual authors and/or other copyright owners. Terms and conditions for use of this material are defined in the policies page.

\section{CLoK}

Central Lancashire online Knowledge www.clok.uclan.ac.uk

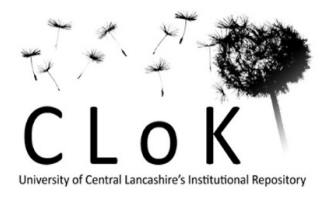




\title{
Analytical Methods
}

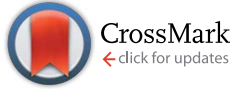

Cite this: DOI: 10.1039/c5ay00502g

\section{Investigating optimum sample preparation for infrared spectroscopic serum diagnostics}

\author{
Lila Lovergne, ${ }^{\text {ab }}$ Graeme Clemens, ${ }^{a}$ Valérie Untereiner, ${ }^{\text {bc }}$ Roman A. Lukaszweski, ${ }^{d}$ \\ Ganesh D. Sockalingum $\dagger^{\mathrm{b}}$ and Matthew J. Baker† ${ }^{\star a}$
}

Biofluids, such as serum and plasma, represent an ideal medium for the diagnosis of disease due to their ease of collection, that can be performed worldwide, and their fundamental involvement in human function. The ability to diagnose disease rapidly with high sensitivity and specificity is essential to exploit advances in new treatments, in addition the ability to rapidly profile disease without the need for large scale medical equipment (e.g. MRI, CT) would enable closer patient monitoring with reductions in mortality and morbidity. Due to these reasons vibrational spectroscopy has been investigated as a diagnostic tool and has shown great promise for serum spectroscopic diagnostics. However, the optimum sample preparation, optimum sampling mode and the effect of sample preparation on the serum spectrum are unknown. This paper examines repeatability and reproducibility of attenuated total reflection (ATR) compared to transmission sampling modes and their associated serum sample preparation with spectral standard deviation of 0.0015 (post pre-processing) achievable for both sampling modes proving the collection of robust spectra. In addition this paper investigates the optimum serum sample dilution factor for use in high throughput transmission mode analysis with a 3-fold dilution proving optimum and shows the use of ATR and transmission mode spectroscopy to illuminate similar discriminatory differences in a patient study. These fundamental studies provide proof of robust spectral collection that will be required to enable clinical translation of serum spectroscopic diagnostics.

Received 25th February 2015 Accepted 21st March 2015

DOI: 10.1039/c5ay00502g

www.rsc.org/methods

\section{Introduction}

Rapid detection of diseases enables the early administration of a therapeutic strategy when the treatment is most effective, thus saving health expenditure and lives. For this purpose Fourier Transform Infrared (FTIR) spectroscopy is a suitable technique as it is non-destructive, label-free, rapid, cost-effective, easy to operate and requires simple sample preparation. Moreover, the use of serum spectroscopy for diagnostics has the advantage for patients to be relatively non-invasive compared to current diagnostic methodologies such as biopsies.

Over the past few years many studies have shown the successful use of vibrational spectroscopy for the analysis of biological samples. ${ }^{\mathbf{1} 2}$ Recent studies have investigated the

\footnotetext{
${ }^{a}$ WESTChem, Department of Pure and Applied Chemistry, University of Strathclyde, Glasgow, G1 1XL, UK. E-mail: matthew.baker@strath.ac.uk; Web: http://www. twitter.com/ChemistryBaker

${ }^{b}$ Université de Reims Champagne-Ardenne, CNRS UMR 7369-MEDyC, Equipe MéDIANBiophotonique et Technologies pour la Santé, UFR de Pharmacie, 51 rue Cognacq-Jay, 51096 Reims Cedex, France

${ }^{c}$ Plateforme Imagerie Cellulaire et Tissulaire, Université de Reims, 51 Cognacq-Jay, 51096 Reims Cedex, France

${ }^{d}$ Biomedical Sciences Department, Defence Science and Technology Laboratory, Porton Down, Salisbury, Wiltshire, SP4 OJQ, UK

$\dagger$ Both authors contributed equally to this work and project supervision.
}

potential of vibrational spectroscopy as a diagnostic tool for the analysis of biofluids. A pilot study on saliva using FTIR spectroscopy associated with linear discriminant analysis (LDA), has reported the correct classification of diabetic patients from healthy control (overall accuracy of $100 \%$ on the training set and $88.2 \%$ on the validation test). ${ }^{3}$ The potential of human tears for the diagnosis of ocular diseases has been evaluated in preliminary studies, Travo et al. have shown significant discrimination $(p<0.0001)$ of healthy people from patients with keratoconus (degenerative disorder affecting the cornea) and also between patients at an early or advanced stage of disease by high throughput (HT)FTIR and principal component analysis (PCA). ${ }^{4-6}$ A study on synovial fluid by Raman spectroscopy and $k$-means analysis, has shown the discrimination of patients with osteoarthritis of low or high severity ( $74 \%$ sensitivity and $71 \%$ selectivity). ${ }^{7}$ Bile patient samples with malignant biliary strictures were differentiated from samples of patients with benign biliary diseases (sensitivity between $82 \%$ and 95\%, specificity between $85 \%$ and $100 \%$ ) by HT-FTIR spectroscopy in association with support vector machine (SVM) classification and leave-one-out cross validation (LOOCV). ${ }^{8}$ A Raman analysis combined with PCA and quadratic discriminant analysis (QDA) performed on urine has allowed the discrimination of patients suffering from diabetes mellitus and hypertension 
with low, high risk of kidney failure or with kidney failure (under dialysis) with a $70 \%$ overall classification rate $(89 \%$ for the control group and $81 \%$ for both high risk and renal failure groups). ${ }^{9}$ However, the most popular biofluids analysed by vibrational spectroscopy are blood-derived products such as plasma ${ }^{10-14}$ and serum. ${ }^{15-18}$ Proof-of-principle studies have shown the ability of FTIR spectroscopy to identify different types of cancer from serum samples. HT-FTIR spectroscopy in transmission mode, was used to discriminate urinary bladder cancer patients from patients with urinary tract infection with an accuracy between 85 and $92 \%$ achieved with LDA or random forest (RF) classifier, ${ }^{19}$ breast cancer from healthy donors with 96\% sensitivity and 93\% specificity with cluster analysis, ${ }^{20}$ cirrhotic patients with or without hepatocellular carcinoma with $87 \%$ sensitivity and $85 \%$ specificity by SVM-LOOCV. ${ }^{21}$ Studies using attenuated total reflection (ATR)-FTIR spectroscopy, coupled with classification machine analysis obtained an accuracy of $95 \%$ and $82 \%$ for the discrimination of ovarian and endometrial cancer $^{22}$ and allowed to achieve $87.5 \%$ sensitivity and $100 \%$ specificity for the differentiation of glioblastoma multiforme (GBM) versus healthy control as well as on average of $94 \%$ sensitivity and $96.5 \%$ specificity for the diagnosis of low grade gliomas, GBM and healthy control. ${ }^{23,24}$

Vibrational spectroscopy provides information on the composition and structure of matter. The principle of IR spectroscopy is based on the interaction of IR light with matter. Molecular bonds absorb the IR radiation at the resonant frequency of the bond or group, exciting vibrational modes. The resultant spectrum is a biochemical fingerprint of the analysed sample, each absorption peak/band corresponds to a specific vibration or combination of vibrations of a molecular bond. This absorption phenomenon obeys the Beer-Lambert law, thus allowing to obtain both quantitative and qualitative information. ${ }^{25}$ In order to realise the full potential of IR spectroscopy as a leading healthcare tool, some issues need to be understood prior to clinical translation. ${ }^{26}$ One issue in particular related to biofluid spectroscopy is due to the strong IR activity of water. As such, the most common protocol for analysing liquids such as biofluids is the drying of drop deposits. However, it has been shown by optical and spectroscopic assessment that this deposition is not homogenous. ${ }^{27-31}$ Thus, reproducibility and reliability of drop-dried spectroscopic results are still questioned. This is even more pronounced when using microspectroscopy. In addition, when analysing biofluids with IR transmission mode it is common to dilute the sample, due to saturation of spectral peaks, with water prior to drying. The effect upon the spectrum and the optimum dilution is unknown and has not been previously investigated. In order to take full advantage of the potential of biofluid spectroscopy to provide a rapid and responsive diagnostic environment, this study reports the use of HT-FTIR, FTIR imaging and ATR-FTIR to investigate and understand the effect of sample preparation upon the IR spectrum and provide an optimum sample preparation for biofluid spectroscopy.

\section{Materials and methods}

\section{Serum samples}

Normal human mixed pooled serum purchased from TCS Biosciences (UK) were used for dilution tests. Serum samples $(n=105)$ from 70 patients needing high risk surgery (e.g. liver and renal surgery, lung resection, large bowel resection, gastrectomy, Whipple's procedure) were provided by the Defence Science \& Technology Laboratory (Dstl) Porton Down. The study has been approved by Southampton \& South West Hampshire Research Ethics Committee (A), $1^{\text {st }}$ Floor, Regents Park Surgery, Shirley, Southampton, Hants, SO16 4RJ (reference number 06/Q1702/152). A consent form was signed by all patients recruited into the study at the medical care centres involved (Liverpool Royal and University Hospital, University College Hospital, London, Bristol Royal Infirmary, Guy's \& St Thomas' Hospital, London, Birmingham Queen Elizabeth Hospital and University Hospital, Frankfurt). Clotted blood samples $(4 \mathrm{~mL})$ were centrifuged and the extracted serum samples were stored at $-80{ }^{\circ} \mathrm{C}$ until needed for analysis. The sera collected include 35 pre-operative samples, 35 postoperative samples from the same patients the day following surgery and 35 additional pre-operative samples from different patients. The study included 35 males and 32 females (the gender of 3 patients from records were not provided) between 21 and 79 years old with an average age of 60 years.

\section{Sample preparation for HT-FTIR and FTIR imaging}

Four dilutions $(1 / 2,1 / 3,1 / 4,1 / 5)$ were performed on normal pooled serum with physiological water $(\mathrm{NaCl} 0.9 \%$, same salinity as blood and used for intravenous injection and dilution of drugs purchased from B. Braun, France). Five microliters per replicate and 10 replicates per sample including diluted, non-diluted normal serum and physiological water as control, were pipetted onto each well of a silicon plate (384 wells). Drops were dried at room temperature before the spectral data acquisition. Serum samples from patients were 3 -fold diluted and 8 replicates were prepared using the same protocol.

\section{Infrared spectroscopy and imaging}

Spectra of diluted, non-diluted pooled serum, physiological water ( 30 spectra per condition, 180 spectra in total) and patient serum samples (840 spectra in total) were collected with a highthroughput screening HTS-XT extension coupled to a Tensor 27 spectrometer (both from Bruker Optics GmbH, Ettlingen, Germany). Spectral acquisition was performed in the transmission mode, controlled via OPUS v6.5 software (Bruker Optics $\mathrm{GmbH}$, Ettlingen, Germany) and collected at a spectral resolution of $4 \mathrm{~cm}^{-1}$, co-adding 32 scans over the spectral range of 4000-400 $\mathrm{cm}^{-1}$. A background spectrum was acquired before each sample measurement and automatically removed from sample spectra. One spectrum is obtained over the whole surface of a well ( $\sim 4 \mathrm{~mm}$ diameter) of the 384 well silicon plate used in the HTS-XT extension. 

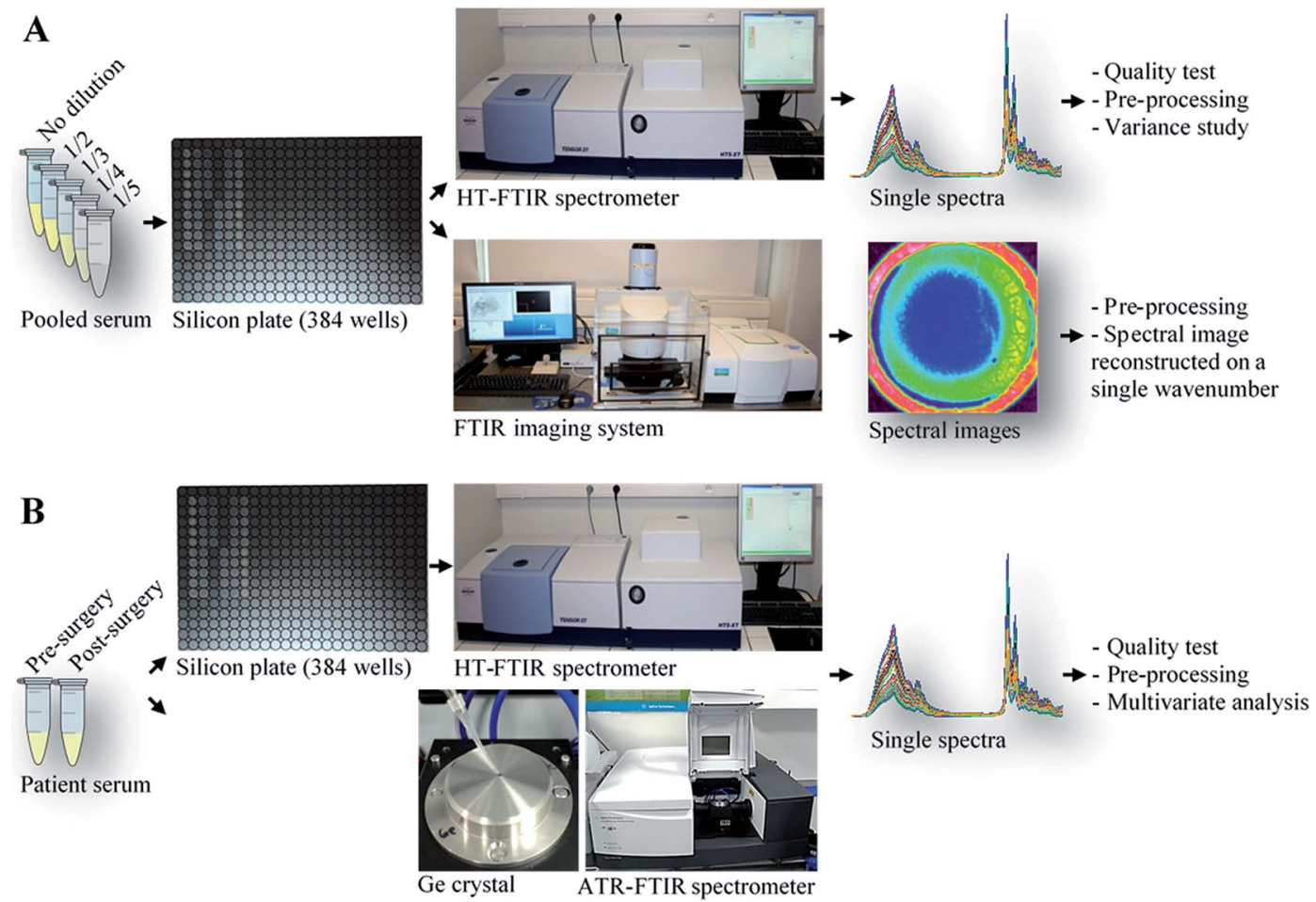

Fig. 1 Schematic methodology representation. (A) Dilution test study performed on human pooled serum by high throughput-Fourier transform infrared (HT-FTIR) spectroscopy and FTIR imaging. (B) HT-FTIR and total attenuated reflection (ATR)-FTIR spectroscopy comparison study performed on pre- $(n=70)$ and post- $(n=35)$ surgery patient serum samples. Ge: germanium.

To study the drop heterogeneity, spectral images of the diluted serum drops were acquired with a PerkinElmer Spectrum Spotlight 400 imaging system (Courtaboeuf, France). Three spectral images were performed on non-diluted, diluted pooled serum and physiological water (18 spectral images in total) at a spatial resolution of $25 \mu \mathrm{m}$ per pixel, a spectral resolution of $4 \mathrm{~cm}^{-1}, 8$ co-added scans, and covering the spectral range of $4000-750 \mathrm{~cm}^{-1}$ (Spectrum-Image software, version 1.6, PerkinElmer).

Patient serum sample spectra were also collected using a Cary 600 series FTIR spectrometer from Agilent technologies, USA, equipped with a Pike Miracle ATR Accessory. After thawing, $1 \mu \mathrm{L}$ of each sample was pipetted onto a germanium (Ge) crystal and dried for 8 minutes at room temperature. Three spectra were collected per biological replicate and 3 replicates performed for each sample ( 9 spectra per patient sample and 945 spectra for all samples in total). Spectra were acquired at a resolution of $4 \mathrm{~cm}^{-1}$, over the spectral range of $4000-400 \mathrm{~cm}^{-1}$ and averaged over 32 co-added scans (Agilent software, Agilent technologies, USA). A background absorption spectrum of the crystal was measured for atmospheric correction before each replicate sample analysis and removed automatically from every sample spectrum. One average spectrum is obtained over the whole surface of the ATR crystal in contact with a sample. The crystal was washed between each replicate analysis. Experimental processes are detailed in the experimental schematic shown in Fig. 1.

\section{Pre-processing and data analysis}

A quality test as previously described ${ }^{21}$ was performed on raw spectra to control absorbance intensity, signal-to-noise ratio and water vapour content (OPUS v6.5 software, Bruker Optics $\mathrm{GmbH}$, Ettlingen, Germany). Spectra with a maximum absorbance higher than 1.8 or lower than 0.35 in arbitrary units (a.u.) were rejected. Signal-to-noise ratio was calculated taking the maximum absorbance of the Amide I band between 1700 and $1600 \mathrm{~cm}^{-1}(S 1)$ and between 1260 and $1170 \mathrm{~cm}^{-1}(S 2)$ respectively divided by the noise intensity evaluated between $2100-$ $2000 \mathrm{~cm}^{-1}(N)$. Spectra were rejected when $S 1 / N$ was less than 50 and $S 2 / N$ was less than 10 . Water vapour content $(W)$ was evaluated between the $1847-1837 \mathrm{~cm}^{-1}$. Spectra were rejected when $S 1 / W$ was less than 20 and $S 2 / W$ was less than 4 .

Following the quality test, data reproducibility was assessed on both raw and pre-processed spectra of diluted normal pooled serum via a variance study which consists in calculating the mean spectrum and the standard deviation (STD) for each sample dilution condition (Fig. 3). Pre-processing of HT-FTIR serum dilution test spectra was performed with OPUS v6.5 software (Bruker Optics GmbH, Ettlingen, Germany). Spectra were cut to the spectral range of $4000-900 \mathrm{~cm}^{-1}$, base-line corrected and vector normalised.

Spectral images of serum drops were first subjected to an atmospheric correction for water vapour and $\mathrm{CO}_{2}$ by the built-in Spectrum-Image software V1.6 (PerkinElmer, Courtaboeuf, France) of the Spotlight 400 imaging system. Spectral images were then reconstructed, based on a specific chosen single 
wavenumber in order to study the wavenumber-related component distribution within drops (Spectrum-Image V1.6).

HT-FTIR and ATR-FTIR patient data pre-processing and multivariate analysis were realised with Matlab software version 7.11.0 (The Math Works, Inc., USA). All spectra were cut to the spectral range of $1800-900 \mathrm{~cm}^{-1}$ (fingerprint region). A principal component based noise reduction using the first 30 principal components (PCs) of the data was performed on the spectra to improve the signal-to-noise ratio. All spectra were then base-line corrected and vector normalised. Principal Component-Discriminant Function Analysis (PC-DFA) was performed on pre-processed data over the spectral region of $1200-950 \mathrm{~cm}^{-1}$.

\section{Results and discussion}

\section{Serum dilution effect}

Four dilutions (2-fold, 3-fold, 4-fold and 5-fold) of serum samples were prepared with physiological water in order to determine the most appropriate dilution for spectral acquisition with HT-FTIR spectroscopy as resulting spectra of nondiluted serum samples were saturated (Fig. 2) at the protein Amide I and Amide II bands (1700-1500 $\mathrm{cm}^{-1}$ ) and $\mathrm{H}-\mathrm{O}-\mathrm{H}$ stretching band at $3280 \mathrm{~cm}^{-1}$. In the 2 -fold dilution sample group, $60 \%$ of the raw spectra failed the quality test, mainly due to a high maximum absorbance ( $>1.8$ a.u.) observed in the Amide I band. On the contrary with a 5-fold dilution, $10 \%$ of the

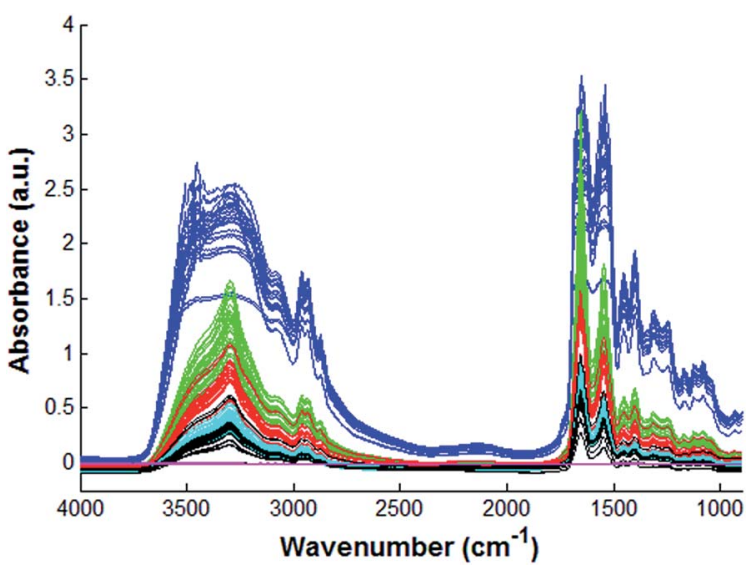

Fig. 2 HT-FTIR raw spectra of non-diluted human pooled serum (blue), diluted samples (2-fold: green; 3-fold: red; 4-fold: cyan; 5-fold: black) and physiological water as negative control (pink). spectra were rejected because of a too low maximum absorbance $(<0.35$ a.u.) of the Amide I band. With the 3 - and 4 -fold dilution, all spectra passed the quality test with a maximum absorbance of 1.6 and 0.9 respectively.

A variance study on both raw spectra and pre-processed spectra was carried out on the spectra that passed the quality test. Standard deviations (STD) of spectra from each dilution (raw and pre-processed) are reported in Table 1. Pre-processed spectra presented a lowest average STD compared to their corresponding raw data (Fig. 3). The pre-processing has improved the reproducibility of the spectra. The 2-fold diluted pre-processed spectra showed the lowest average STD due to the low number of remaining spectra after the quality test (40\%). The 5-fold diluted pre-processed spectra presented the highest average STD (0.0022). The STD results of the 3 and 4 fold dilution are similar with both providing low average STD after preprocessing (0.0012 and 0.0013 respectively). Globally, the results show a good reproducibility of the spectra, similar to the reproducibility of human pooled serum spectra reported by Hands et al. via ATR-FTIR spectroscopy (average STD of 0.0137 and 0.0015 for raw and pre-processed spectra respectively). ${ }^{24}$

Combining the quality test and the variance study results, the 3-fold dilution was determined as the most suitable dilution for a serum HT-FTIR analysis with a good reproducibility and absorbance intensity. The 3-fold dilution was chosen as a higher dilution (e.g. 4-fold) will induce the risk of a low spectral response, especially for the analysis of molecules present at a low concentration within the serum. No molecular changes were visible on collected spectra, assuming that there was no effect of the dilution with water on the composition of the serum. Similar observations have been reported in a study by Raman spectroscopy on plasma dilution tests. They concluded that the plasma dilution did not affect the chemical composition and molecular structure of proteins within dried drops. ${ }^{30}$

Spectral images were acquired to better understand the distribution of serum molecular components within dried drops as physical differences were observed between nondiluted (presence of cracks) and diluted serum samples (hollow shape) suggesting a drop composition heterogeneity (white light images Fig. 4). It can be seen on the total absorbance spectral image of the droplets that the absorbance intensity varies within the same drop, with a highest absorbance intensity in the drop edge compared to the centre. This translates as a concentration gradient of serum components from the centre to the periphery of the drops, showing an uneven drying process across the serum droplets. As expected a global absorbance

Table 1 Quality test and variance study results performed on HT-FTIR spectra of normal pooled serum with different dilutions

\begin{tabular}{llll}
\hline Dilution & $\begin{array}{l}\text { Right spectra after } \\
\text { quality test (\%) }\end{array}$ & $\begin{array}{l}\text { Mean STD } \\
\text { (raw spectra) }\end{array}$ & $\begin{array}{l}\text { Mean STD } \\
\text { (pre-processed spectra) }\end{array}$ \\
\hline $1 / 2$ & 40 & 0.015 & 0.0004 \\
$1 / 3$ & 100 & 0.0474 & 0.0012 \\
$1 / 4$ & 100 & 0.02 & 0.0013 \\
$1 / 5$ & 90 & 0.0342 & 0.0022
\end{tabular}



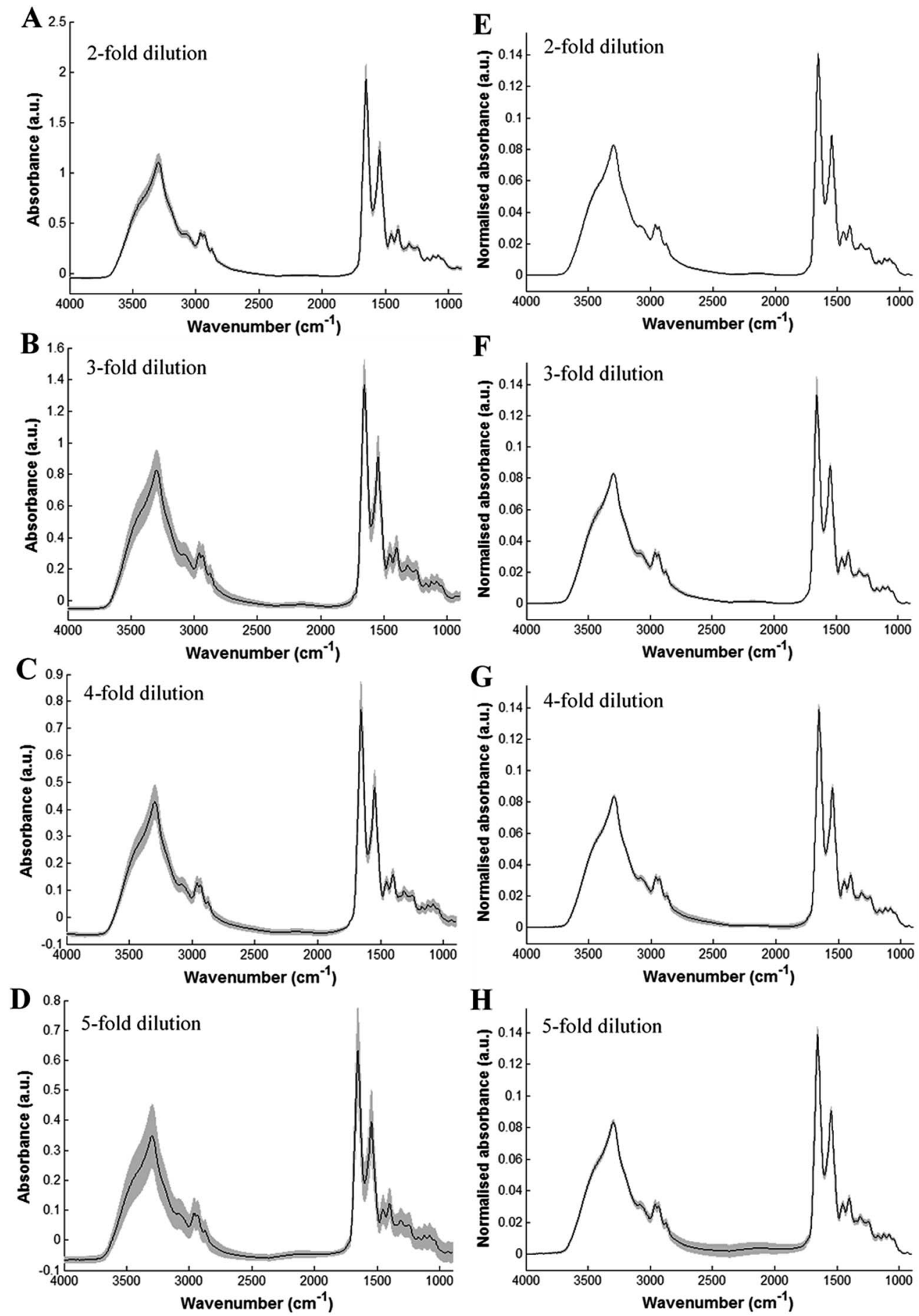

Fig. 3 Variance study performed on HT-FTIR raw (A-D) and pre-processed $(E-H)$ mean spectra (black) of diluted human pooled serum. Standard deviation is represented in grey.

intensity decrease was observed for diluted samples compared to non-diluted samples (total absorbance spectral images Fig. 4). Spectral images based on a single wavenumber reconstruction enabled the determination, within a drop, of the distribution of molecules associated with the targeted peak. It was observed (reconstructed spectral images Fig. 4) that serum components such as proteins (Amide I and Amide II bands around $1647 \mathrm{~cm}^{-1}$ and $\left.1542 \mathrm{~cm}^{-1}\right)$, lipids $\left(1442 \mathrm{~cm}^{-1}\right)$ and nucleic acids $\left(1078 \mathrm{~cm}^{-1}\right)$ have a tendency to migrate towards the periphery of the drops. This phenomenon is referenced in the literature as the well-known coffee-ring effect. ${ }^{32-34}$ This effect was more visible when the dilution factor increased, the ring appearing thinner at the drops' edges and the centres appearing more hollowed compared to non-diluted serum samples (white 




Fig. 4 Serum dilution study by FTIR imaging of dried drops. Original spectral images were atmospheric corrected. Other spectral images were reconstructed on a single wavenumber. Abs: absorbance.

light images Fig. 4). The deposit and the resulting drop morphology has also been shown to be influenced by the substrate hydrophobicity but without impact on the chemical composition of samples. ${ }^{30}$ To avoid the coffee-ring effect, a study has recommended to perform an analysis on a film composed of reduced-size drops ( 217 drops of $200 \mathrm{pL}$ each per 

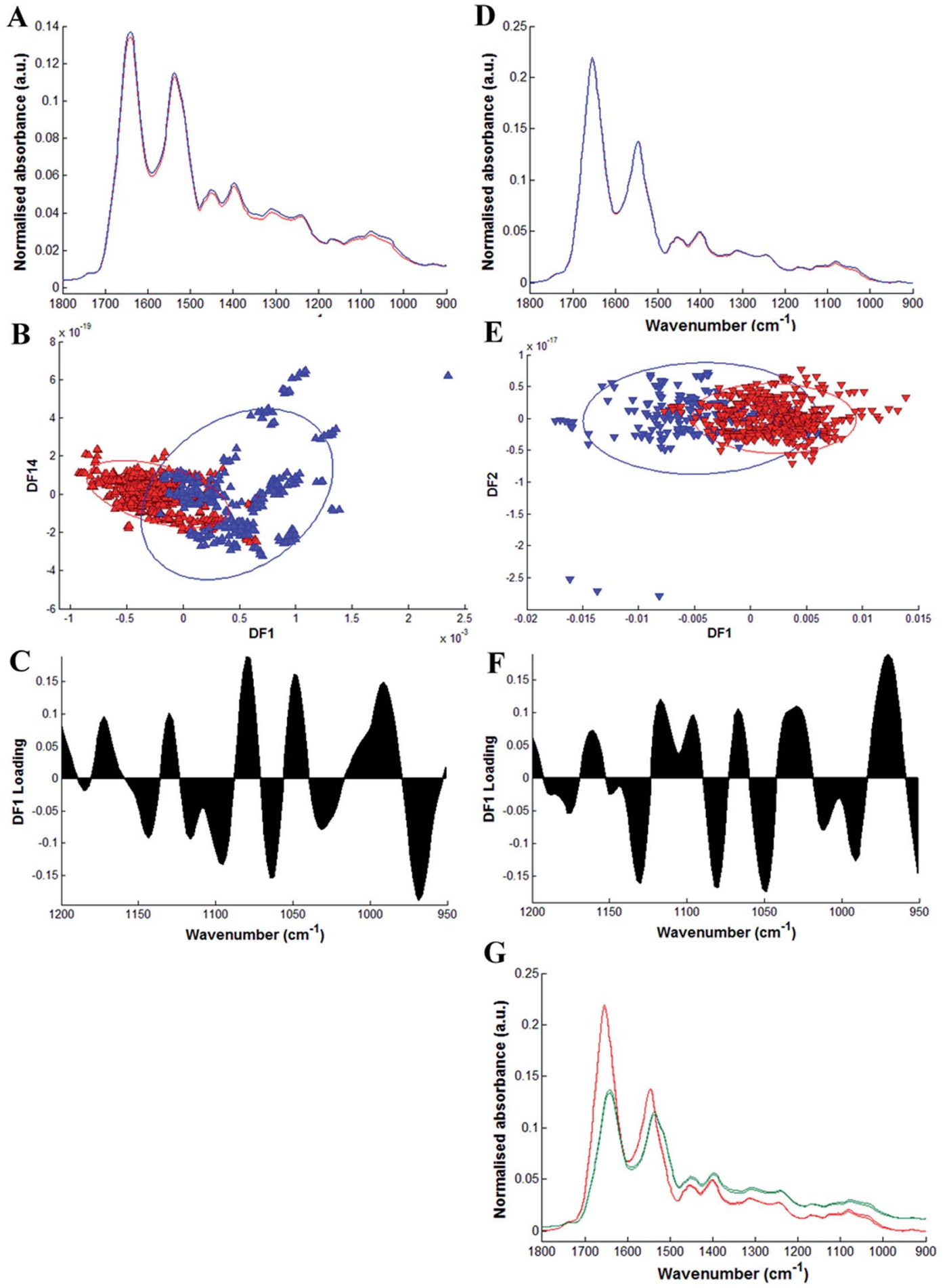

Fig. 5 ATR-FTIR and HT-FTIR comparison study on pre and post-surgery serum samples. (A and D) ATR-FTIR (A) and HT-FTIR (D) pre-processed mean spectra of patient serum prior to surgery in red and after surgery in blue. (B and E) ATR-FTIR (B) and HT-FTIR (E) PC-DFA scatter plot, preprocessed serum spectra of patients prior to surgery are represented by red triangles and after surgery by blue triangles over the spectral range of 950-1200 $\mathrm{cm}^{-1}$ with their respective $90 \%$ confidence ellipses. (C and F) ATR-FTIR (C) and HT-FTIR (F) corresponding DF1 loading from whole patient serum. (G) ATR-FTIR and HT-FTIR spectra (from A and D) in green and red respectively.

well of a 384 wells silicon plate) obtained by an automatic sampling and by controlling the drying process (vacuum drying). They obtained a higher reproducibility of spectral data compared to a non-automatic sampling. ${ }^{35}$
The presence of cracks has been observed on non-diluted sample drops and was less visible/absent on diluted samples (white light images Fig. 4). It has been reported that spectra acquired from sample areas containing cracks suffer from 
spectral distortions resulting in an abnormal Amide I and II bands and baseline. ${ }^{29}$ In this study, the dilution of the serum samples has provided visually a dried drop with greater homogeneity over the surface. Moreover, one averaged spectrum is acquired on the whole surface of a well with the HT-FTIR technique, resulting in a representative spectrum of the entire serum sample contrary to spectra collected in a single point mode as serum dried drops spectra have shown spatial but also chemical heterogeneity. ${ }^{28}$

\section{ATR-FTIR and HT-FTIR spectroscopy comparison}

An analysis was carried out on pre- and post-operative serum samples from patients having undergone surgery for different clinical reasons. This set of samples $(n=105)$ was used to compare the two techniques ATR-FTIR and HT-FTIR spectroscopy and also to enable the profiling of a baseline IR response when considering only surgery. ATR-FTIR is an excellent technique for the analysis of serum since a sample can be directly deposited and dried on the ATR crystal before the spectral acquisition. ${ }^{36}$ However, there are currently no multi-well sample accessories to enable rapid automated collection of spectra. HT-FTIR enables the rapid automated collection of spectra but there is an increased requirement for further sample preparation via sample dilution. As such it takes 6 hours to collect 360 spectra from 360 samples on a multi-well plate via HT-FTIR spectroscopy and in the same amount of time 40 spectra would have been able to be collected via the current ATR-FTIR approach (the addition sample preparation time for the HTFTIR takes approximately 1 hour for the deposit of 360 samples and 30 minutes for the samples drying). The sample preparation regarding the analysis of serum by ATR-FTIR has been reproduced from the methodology applied by Hands et $a .^{24}$ They have determined that a serum drop of $1 \mu \mathrm{L}$ is dry after 8 minutes at room temperature and that the resultant spectra acquired exhibited a high reproducibility. In the present study, spectra from human serum samples were collected from $1 \mu \mathrm{L}$ and $5 \mu \mathrm{L}$ (3-fold diluted serum) for the ATR-FTIR and HT-FTIR spectroscopy respectively. These spectra were reproducible and repeatable thus conserving the serum resource by using minute amounts. Spectral differences are visible on the average spectra of the two pre- and post-operative sample groups especially between 950 and $1200 \mathrm{~cm}^{-1}$ for both ATR-FTIR and HT-FTIR techniques, further differences are discernable between 1250 and $1700 \mathrm{~cm}^{-1}$ but only with the ATR-FTIR method (Fig. 5A and D). A different ratio and a peak shift is observable for the Amide I and Amide II peaks between ATR and transmission sampling modes (Fig. 5A, D and 5G). This is similar to the ratio and peak shift differences observed by Ollesch et al. ${ }^{35}$ on dried serum sample comparison between ATR and transmission due to dispersion effects. Associated with PC-DFA, both ATR-FTIR and HT-FTIR spectroscopies show similar scores plots and discriminatory ability to separate serum spectra over the range of $1200-950 \mathrm{~cm}^{-1}$ of patients prior to surgery and serum spectra after surgery in spite of similarities resulting in an overlap. Discriminant Function 1 (DF1) in both cases is responsible for the separation of the two spectral groups

\begin{tabular}{|c|c|}
\hline Frequency $\left(\mathrm{cm}^{-1}\right)$ & Assignments \\
\hline 1170 & Ester $\mathrm{C}-\mathrm{O}$ asymmetric stretch \\
\hline 1150 & C-O stretch (carbohydrates) \\
\hline 1125 & $\mathrm{C}-\mathrm{C}$ asymmetric stretch \\
\hline 1110 & $\mathrm{CN}$ stretch, $\mathrm{CH}$ bend \\
\hline 1100 & $\mathrm{PO}_{2}^{-}$symmetric stretch (nucleic acid) \\
\hline 1078 & C-O stretch (deoxyribose/DNA-RNA) \\
\hline 1065 & Chain $\mathrm{C}-\mathrm{C}$ \\
\hline 1050 & $\mathrm{C}-\mathrm{O}$ stretch \\
\hline $1023-1040$ & Glycogen \\
\hline 1006 & Phenylalanine (ring breathing) \\
\hline 990 & $\mathrm{CO}-\mathrm{O}-\mathrm{C}$ stretch \\
\hline 970 & $\mathrm{C}-\mathrm{C}, \mathrm{CN}$ stretch, $\mathrm{PO}_{3}{ }^{2-}$ stretch (DNA) \\
\hline
\end{tabular}

(Fig. 5B and E) and DFs along the $y$-axes are only shown for visual purposes. Loadings on DF1 contain only the spectral information that is responsible for the discrimination between spectral groups (Fig. 5C and F). With both techniques, the same peaks have been identified but have opposite directions on the loadings plots. Positive peaks in DF1 loadings for the ATR-FTIR spectroscopy analysis are negative in the DF1 loadings for the HT-FTIR spectroscopy study and vice versa. Spectra from the two groups are also separated in an inverse way against the $x$-axis of the scatter plots between the two techniques. Proposed peaks assignments are presented in Table 2. Major peaks of the loadings on DF1 were in both cases $1040-1023 \mathrm{~cm}^{-1}$ corresponding to glycogen and $1078 \mathrm{~cm}^{-1}$ assigned as $\mathrm{C}-\mathrm{O}$ stretch (DNA/RNA). It can be noted that a complete separation of the two groups by PC-DFA was not expected since it is known that a surgical procedure triggered physiological and immunological reactions in order to maintain the body homeostasis but the stress induced by surgery and the associated body responses are dependent of many factors such as the kind of procedure and its relative injury severity, pre-existing pathologies, genetic predisposition of patients and the difference observed will not be as significant as diseased versus non-diseased patient samples. ${ }^{3-39}$

\section{Conclusion}

HT-FTIR and ATR-FTIR techniques have both shown promising results for serum dried-drops analysis with a high reproducibility of the spectra collected on normal human pooled mixed serum samples. Moreover, both approaches associated with multivariate analysis have shown an equivalent capability to distinguish spectra of patient serum collected before and after surgical procedures. This basal IR response to surgery should be taken into consideration when performing a diagnostic study as molecular changes observed in spectra might be due to the surgery/inflammation itself and not to the pathology targeted, however this study has shown that these responses are not significant enough to enable full differentiation of serum samples. Compared to the HT-FTIR technique where serum samples have to be diluted because of a saturation effect, with 
the ATR-FTIR method, samples can be directly applied onto the ATR crystal and can be portable. However, this implies no possible sample storage for further analysis as the crystal has to be cleaned after each sample measurement and no automation of the analysis is yet available. Regarding these aspects, with the HT-FTIR technique the storage of samples is in theory feasible but no studies have investigated the effect of the conservation over the time of serum properties integrity. A 3-fold dilution was shown to be optimum based upon a variance study, quality test and the ability to detect low concentration biochemicals present compared to higher dilutions and is the recommendation for HT-FTIR analysis. HT-FTIR spectroscopy has the advantage to allow an automated analysis that is an important criterion for a transposition of IR spectroscopy into a clinical application. This automation advantage can be seen in the time comparison with HT-FTIR being able to collect 360 spectra, in the same time it would allow to collect 40 spectra in the current ATR-FTIR approach. An automated approach for ATR-FTIR analysis would be beneficial as there is a comparable spectral reproducibility between ATR-FTIR and HT-FTIR spectroscopy and a comparable ability to discriminate and illuminate differences in patient spectra but with an automated ATR-FTIR approach, there would not be a need for dilution.

\section{Acknowledgements}

The authors would like to acknowledge the support of the Defence Science \& Technology Laboratory (Dstl) and the Direction Générale de l'Armement (DGA). MJB, GS, RAL and LL acknowledge the Defence Science \& Technology Laboratory (Dstl) Anglo-French PhD Scheme for funding (Contract no. DSTLX-1000084129).

\section{Notes and references}

1 M. J. Baker, J. Trevisan, P. Bassan, R. Bhargava, H. J. Butler, K. M. Dorling, P. R. Fielden, S. W. Fogarty, N. J. Fullwood, K. A. Heys, C. Hughes, P. Lasch, P. L. Martin-Hirsch, B. Obinaju, G. D. Sockalingum, J. Sulé-Suso, R. J. Strong, M. J. Walsh, B. R. Wood, P. Gardner and F. L. Martin, Nat. Protoc., 2014, 9, 1771-1791.

2 D. I. Ellis and R. Goodacre, Analyst, 2006, 131, 875-885.

3 D. A. Scott, D. E. Renaud, S. Krishnasamy, P. Meriç, N. Buduneli, S. Çetinkalp and K. Liu, Diabetol. Metab. Syndr., 2010, 2, 48-56.

4 J. Filik and N. Stone, Anal. Chim. Acta, 2008, 616, 177-184.

5 C. Lin, K. Ming-Tse and C. Hsien-Chang, J. Med. Biol., 2010, 30, 343-354.

6 A. Travo, C. Paya, G. Déléris, J. Colin, B. Mortemousque and I. Forfar, Anal. Bioanal. Chem., 2014, 406, 2367-2376.

7 K. A. Esmonde-White, G. S. Mandair, F. Raaii, J. A. Jacobson, B. S. Miller, A. G. Urquhart, B. J. Roessler and M. D. Morris, J. Biomed. Opt., 2009, 14, 034013.

8 V. Untereiner, G. D. Sockalingum, R. Garnotel, C. Gobinet, F. Ramaholimihaso, F. Ehrhard, M. Diebold and G. Thiéfin, J. Biophotonics, 2014, 7, 241-253.
9 J. A. Bispo, E. E. De Sousa Vieira, L. Silveira and A. B. Fernandes, J. Biomed. Opt., 2013, 18, 087004.

10 V. Erukhimovitch, M. Talyshinsky, Y. Souprun and M. Huleihel, Vib. Spectrosc., 2006, 40, 40-46.

11 K. Singh, K. Lee, D. Lee, Y. K. Kim and K. C. Kim, J. Mech. Sci. Technol., 2010, 24, 1661-1668.

12 K. W. C. Poon, F. M. Lyng, P. Knief, O. Howe, A. D. Meade, J. F. Curtin, H. J. Byrne and J. Vaughan, Analyst, 2012, 137, 1807-1814.

13 U. Neugebauer, S. Trenkmann, T. Bocklitz, D. Schmerler, M. Kiehntopf and J. Popp, J. Biophotonics, 2014, 7, 232-240.

14 C. Lacombe, V. Untereiner, C. Gobinet, M. Zater, G. D. Sockalingum and R. Garnotel, Analyst, 2015, 140, 2280-2286.

15 J. L. Pichardo-Molina, C. Frausto-Reyes, O. Barbosa-García, R. Huerta-Franco, J. L. González-Trujillo, C. A. RamírezAlvarado, G. Gutiérrez-Juárez and C. Medina-Gutiérrez, Laser. Med. Sci., 2007, 22, 229-236.

16 W. Petrich, K. B. Lewandrowski, J. B. Muhlestein, M. E. H. Hammond, J. L. Januzzi, E. L. Lewandrowski, R. R. Pearson, B. Dolenko, J. Früh, M. Haass, M. M. Hirschl, W. Köhler, R. Mischler, J. Möcks, J. Ordóñnez-Llanos, O. Quarder, R. Somorjai, A. Staib, C. Sylvén, G. Werner and R. Zerback, Analyst, 2009, 134, 1092-1098.

17 E. Scaglia, G. D. Sockalingum, J. Schmitt, C. Gobinet, N. Schneider, M. Manfait and G. Thiéfin, Anal. Bioanal. Chem., 2011, 401, 2919-2925.

18 M. Khanmohammadi, A. B. Garmarudi, M. Ramin and K. Ghasemi, Microchem. J., 2013, 106, 67-72.

19 J. Ollesch, M. Heinze, H. M. Heise, T. Behrens, T. Brüning and K. Gerwert, J. Biophotonics, 2014, 7, 210-221.

20 J. Backhaus, R. Mueller, N. Formanski, N. Szlama, H. G. Meerpohl, M. Eidt and P. Bugert, Vib. Spectrosc., 2010, 52, 173-177.

21 X. Zhang, G. Thiéfin, C. Gobinet, V. Untereiner, I. Taleb, B. Bernard-Chabert, A. Heurgué, C. Truntzer, P. Ducoroy, P. Hillon and G. D. Sosckalingum, Transl. Res., 2013, 162, 279-286.

22 K. Gajjar, J. Trevisan, G. Owens, P. J. Keating, N. J. Wood, H. F. Stringfellow, P. L. Martin-Hirsch and F. L. Martin, Analyst, 2013, 138, 3917-3926.

23 J. R. Hands, P. Abel, K. Ashton, T. Dawson, C. Davis, R. W. Lea, A. J. S. McIntosh and M. J. Baker, Anal. Bioanal. Chem., 2013, 405, 7347-7355.

24 J. R. Hands, K. M. Dorling, P. Abel, K. M. Ashton, A. Brodbelt, C. Davis, T. Dawson, M. D. Jenkinson, R. W. Lea, C. Walker and M. J. Baker, J. Biophotonics, 2014, 7, 189-199.

25 Handbook of instrumental techniques for analytical chemistry, ed. F. A. Settle, Prentice Hall PTR, 1997, ISBN 0-13-177338-0.

26 H. J. Byrne, M. Baranska, G. J. Puppels, N. Stone, B. Wood, K. M. Gough, P. Lasch, P. Heraud, J. Sulé-Suso and G. D. Sockalingum, Analyst, 2015, 140, 2066-2073.

27 J. Filik and N. Stone, Analyst, 2007, 616, 177-184.

28 F. Bonnier, F. Petitjean, M. J. Baker and H. J. Byrne, J. Biophotonics, 2014, 7, 167-179. 
29 C. Hughes, M. Brown, G. Clemens, A. Henderson, G. Monjardez, N. W. Clarke and P. Gardner, J. Biophotonics, 2014, 7, 180-188.

30 K. A. Esmonde-White, F. W. L. Esmonde-White, M. D. Morris and B. J. Roessler, Analyst, 2014, 139, 2734-2741.

31 I. Taleb, G. Thiéfin, C. Gobinet, V. Untereiner, B. BernardChabert, A. Heurgué, C. Truntzer, P. Hillon, M. Manfait, P. Ducoroy and G. D. Sockalingum, Analyst, 2013, 138, 4006-4014.

32 R. D. Deegan, T. F. Dupont, G. Huber, S. R. Nagel and T. A. Witten, Nature, 1997, 389, 827-829.

33 W. Ristenpart, P. Kim, C. Domingues, J. Wan and H. Stone, Phys. Rev. Lett., 2007, 99, 234502.

34 P. J. Yunker, T. Still, M. A. Lohr and A. G. Yodh, Nature, 2011, 476, 308-311.
35 J. Ollesch, S. L. Drees, H. M. Heise, T. Behrens, T. Brüning and K. Gerwert, Analyst, 2013, 138, 4092-4102.

36 K. M. Dorling and M. J. Baker, Trends Biotechnol., 2013, 31, 437-438.

37 E. Lin, S. E. Calvano and S. F. Lowry, Surgery, 2000, 127, 117126.

38 P. V. Giannoudis, H. Dinopoulos, B. Chalidis and G. M. Hall, Injury, 2006, 37S, S3-S9.

39 T. Gutierrez, R. Hornigold and A. Pearce, Surgery, 2011, 29, 93-96.

40 G. L. Owens, K. Gajjar, J. Trevisan, S. W. Fogarty, S. E. Taylor, B. Da Gama-Rose, P. L. Martin-Hirsch and F. L. Martin, J. Biophotonics, 2014, 7, 200-209.

41 F. Bonnier, M. J. Baker and H. J. Byrne, Anal. Methods, 2014, 6, 5155-5160. 